\title{
La Valorisation de la Recherche dans les Petites et
} Moyennes Universités au sein d'un Système d'Innovation : Le cas de l'Université Picardie Jules Verne (UPJV)

\section{The valorisation of research of small and medium-sized universities within an innovation system}

\author{
Vanessa Casadella ${ }^{1}$, Sofiane Tahi $^{2}$ \\ ${ }^{1}$ Université Picardie Jules Verne, Réseau de Recherche sur l'Innovation, France, vanessa.casadella@u-picardie.fr \\ ${ }^{2}$ Université Picardie Jules Verne, France, Réseau de Recherche sur l'Innovation, sofiane.tahi@u-picardie.fr
}

RÉSUMÉ. Concept souple et peu récent, le Système d'Innovation (S.I) sert de référence dans l'orientation des politiques d'innovation et/ou de Science et Technologie (S\&T). II a toutefois été moins utilisé dans l'interrelation entre les systèmes productifs et éducatifs. La valorisation de la recherche permet de nouer les liens Université-entreprises en rendant utilisables ou commercialisables les résultats, les connaissances et les compétences de la recherche. Notre article met en évidence la question de la valorisation de la recherche à l'Université Picardie Jules Verne (UPJV) et son impact au sein du système régional d'innovation. Quelles sont les formes de sa valorisation dans le cadre de la Petite et Moyenne Université (P.M.U) française qu'elle représente ? A-t-elle des spécificités liées à sa taille ? En quoi et comment permet-elle de créer et transférer des connaissances avec le secteur productif local et national ? Nous travaillerons sur l'ensemble de ces questions, par une méthodologie reposant à la fois sur des sources secondaires mais surtout sur une enquête quantitative et qualitative réalisée en 2020 auprès des services centraux, VP Recherche et Directeurs de Laboratoire de l'UPJV.

ABSTRACT. As both a flexible and relatively new concept, the Innovation System (IS) serves as a reference in orienting innovation and/or Science and Technology (S\&T) policies. It has, however, been less used in the interrelationship between the productive and educational systems. The valorisation of research makes it possible to forge university-business links by making the results, knowledge and skills of research usable or marketable. Our article highlights the valorisation of university research in the University Picardy Jules Verne (UPJV) and its impact within the Regional Innovation System. What are the forms of its valorization within the framework of the French Small and Medium University (SMU) that it represents? Does it have specificities related to its size? How does it create and transfer knowledge with the local and national productive sector? We will work on all of these questions, using a methodology based on both secondary sources, but above all, on a quantitative and qualitative carried out with central services, VP Research and Laboratory Directors of the UPJV.

MOTS-CLÉS. Système d'Innovation, Valorisation de la Recherche, Petites et Moyennes Universités, Connaissances. KEYWORDS. Innovation System, Valorization of Research, Small and Medium Universities, Knowledge.

Depuis une trentaine d'années, le système d'innovation (S.I) sert principalement de référence dans l'orientation des politiques d'innovation et/ou de science et technologie (S\&T). Il a toutefois été moins utilisé dans l'interrelation entre les systèmes productifs et éducatifs. Or, les systèmes éducatifs sont primordiaux dans les processus d'innovation (Brundenius et al., 2009). D'abord parce qu'ils représentent un acteur clé par les connaissances académiques qu'ils offrent, mais surtout car ils alimentent à travers de multiples collaborations, les systèmes productifs, industriels et commerciaux. Ils permettent à la fois de fournir la main d'œuvre diplômée dans les différents secteurs, tout comme le personnel en R\&D à travers la recherche publique et fondamentale. Le point d'entrée traditionnel qui lie le S.I au système éducatif est celui de l'université, par ses fonctions d'enseignement et de recherche [CON, HEI, 02, CAS, MAT, LAS, 14]. Dans un contexte de valorisation économique des connaissances, les relations «université-entreprise » sont apparues comme un 
moyen privilégié d'optimiser les apports de la science et de la technologie au service du développement économique. Plus précisément, c'est la valorisation de la recherche, qui va permettre de nouer des liens scientifiques et industriels en rendant utilisables ou commercialisables les résultats, les connaissances et les compétences de la recherche. Selon le rapport Guillaume établi en 2007, la valorisation concerne les relations entre les acteurs de la recherche (grands organismes, établissements d'enseignement supérieur...) et le monde économique. La valorisation suppose une mise en relation du monde de la recherche et du monde socio-économique. Elle n'est pas un processus automatique : elle doit être organisée et faire l'objet d'actions concertées et réfléchies. Plus concrètement, elle représente les modalités de transfert de connaissances entre le monde académique et productif. Ces modalités sont importantes pour mesurer le degré de maturité d'un S.I, dont les flux formels et informels sont à la base de son développement et de sa pérennité. En effet, les liens et interactions à l'intérieur et entre les secteurs représentent de véritables flux de connaissances et d'informations et se définissent comme d'importants mécanismes pour le transfert des connaissances tacites et codifiées [NIO, 02].

Si les grandes universités sont souvent étudiées à travers les relations qu'elles entretiennent avec le système productif [MOW, NEL, SAM 01], il en est différemment des petites et moyennes universités (P.M.U), qui, sans définition réellement posée, représentent, selon le rapport Aimé de 2016, les établissements ne faisant pas partie du groupe des universités intensives en recherche et issues de fusions d'établissements réalisées depuis 2008. C'est le cas de l'Université Picardie Jules Verne (UPJV), créée en 1969, et représentant environ 31000 étudiants sur 1,6 millions d'étudiants inscrits dans l'enseignement supérieur en 2018, soit 1,93\% de l'effectif global. Structure moyenne, elle y associe, comme la majorité des P.M.U, des difficultés de nature financière, matérielle et humaine. Mais pour autant, avec 35 unités de recherche, dont 6 associées au CNRS, 2 à l'INSERM et 1 à l'INERIS, la mise en place d'effets de levier par des contrats de recherche régionaux, nationaux et internationaux permet de favoriser la mise en synergie des forces du territoire et d'accroitre la maturité de son système d'innovation. La valorisation de la recherche à l'UPJV est également mise en exergue par des acteurs de transfert tels que la Société d'Accélération de Transfert de Technologie (la SATT Nord) qui facilite les activités de transfert de technologie de la recherche publique vers des entreprises de toute taille, ou des structures associatives comme Ombelliscience, qui œuvrent pour le partage des savoirs dans les domaines scientifiques et techniques. Dans cette perspective, notre article met en évidence la question de la valorisation de la recherche à l'UPJV et son impact au sein du système régional d'innovation dans le cadre d'une petite et moyenne université (PMU) française. Quelles sont les formes de son activité de valorisation ? En quoi et comment permet-elle de créer et transférer des connaissances vers le secteur productif ? Nous travaillerons sur l'ensemble de ces questions, par une méthodologie reposant sur des sources secondaires et une enquête quantitative et qualitative réalisée en janvier 2020. Nous abordons tout d'abord le lien entre le S.I et l'université (§1), puis nous caractérisons la valorisation de la recherche au sein de ce S.I ( 2$)$ et enfin, nous décrivons le cas concret de l’UPJV ( $\$ 3)$ et ses effets potentiels sur le système régional d'innovation qui l'entoure (\$4).

\section{L'importance des universités dans un système d'innovation (S.I)}

L'approche par les systèmes d'innovation n'est pas récente et son cadre d'analyse a été marqué par une grande diversité de ses délimitations empiriques. Avec la multiplication de concepts marqués par la dimension locale, régionale ou sectorielle de l'innovation, le système d'innovation place au cœur du débat sur la dimension des politiques publiques technologiques. Mais le S.I n'a pas été le seul concept discuté dans la littérature économique pour mettre en avant les interrelations sources d'externalités, d'autres ont trouvé légitimité avec les apprentissages localisés des réseaux d'innovation [DJE, GAL, 18], les clusters [SUI, VIC, 2015], les dynamiques sectorielles [GAL, NAD, 16] ou toutes les autres configurations spatiales créatrices d'externalités de connaissances. 
Si la délimitation nationale a davantage trait aux politiques publiques, culture et identité nationales, le territoire régional semble plus adéquat à mobiliser dans le cadre de la valorisation de la recherche au sein d'une université. Un système régional d'innovation (S.R.I) implique des collaborations dans les processus d'innovation entre les entreprises et les organisations créatrices et à l'origine de la diffusion de connaissances telles que les universités, les laboratoires et instituts, les unités de transfert technologique, les associations d'affaires ainsi que les agences financières [DOL, BIT, 05]. Apparue début des années 1990 avec notamment les travaux de Cooke [92], la prise en compte des facteurs régionaux dans les dynamiques industrielles régionales est aujourd'hui à la fois une évidence et une nécessité pour l'analyse et la compréhension des régions innovatrices. Dans ce système régional, l'innovation est un processus territorialisé, organisé à la fois par les ressources localement ancrées, mais également par le contexte social et institutionnel dans lequel elle évolue. En effet, chaque institution joue un rôle spécifique par la distribution des procédures conformes et pratiques standards lesquelles structurent les rapports entre individus. Par ailleurs, les institutions évoluent sans être statiques et sans cadre institutionnel optimal. Ces dernières sont toutefois spécifiques aux régions en question et leurs configurations dépendent des contextes politiques, sociaux, culturels [COO, URA, UTX, 01]. Les organisations, quant à elles, se trouvent enchâssées au sein d'un environnement institutionnel spécifique. Ces dernières se définissent comme des systèmes structurés et institutionnalisés construits pour exécuter un certain nombre de tâches. Elles représentent entre autres, les entreprises, centres de recherches et Universités. Ce sont ces organisations représentées par les universités qui nous intéressent plus particulièrement.

Dans la perspective d'une approche «large» d'un S.I représenté par les institutions et organisations formelles et informelles, un certain nombre de travaux a formalisé l'entrée des systèmes éducatifs comme composantes actives des S.I [LUN, CHR, 99]. Le rôle des universités a par contre été assez écarté avant d'être remis plus récemment dans les débats actuels des travaux en économie de l'innovation avec les modèles de quadruple et quintuple hélice au cœur des S.I [CAR, CAM, 17] ou de l'université entrepreneuriale [SCH, 19].

Le système universitaire dans le S.I est fondamental sur un double aspect : il place les diplômés dans les systèmes productifs (d'où l'importance des marchés du travail) tout comme il fournit le personnel en $R \& D$ à travers la recherche fondamentale. Tout contact Université-entreprise peut également favoriser la création d'entreprises privées issue de la recherche universitaire [BRU, GOR, AGR, 11]. Les collaborations entre universités et entreprises facilitent l'intégration des docteurs au sein des entreprises à partir de la création de projets communs et permettent également une meilleure connaissance en matière de formation professionnelle. Les universités créent des diplômes de plus en plus professionnalisants permettant une meilleure insertion sur le marché du travail. L'objectif des universités est de faire émerger des liens avec le secteur industriel à travers des flux d'informations et de connaissances.

Selon les régions étudiées, un certain nombre de caractéristiques liées à la R\&D et l'innovation sont présentes [CAS, MAT, LAS, 14] :

- Les entreprises sont davantage engagées dans des dépenses liées à la $R \& D$ interne plutôt qu'à la $\mathrm{R} \& \mathrm{D}$ externe,

- Les entreprises privilégient les sources internes pour innover,

- Seule une petite proportion d'entreprises considère les Universités comme une importante source d'innovation et d'informations.

Tous les systèmes universitaires ne se situent pas dans la même configuration en termes de ressources techniques, matérielles et financières. Dans certains pays en développement, les universités sont particulièrement vulnérables : privatisation, faible qualité de l'enseignement public, 
infrastructures dégradées, faibles salaires d'enseignants-chercheurs. Leur détériotation est notoire malgré un rôle primordial dans le développement économique. Mais d'autres universités sont mieux armées pour se construire et transférer des connaissances au sein de leurs systèmes d'innovation. Elles jouent un rôle déterminant dans le développement de territoires consacrés aux technologies de pointe à travers la production de connaissances, la formation de main d'œuvre et la création d'entreprises par essaimage [CAS, HAL, 94]. Dans certaines régions, elles facilitent les processus d'innovation et d'apprentissage. La croissance rapide de l'économie infrarégionale aux États-Unis, comme dans le comté de Santa Clara en Californie (Silicon Valley) grâce à la contribution de l'Université Stanford, ou à Boston et ses environs grâce à celle du Massachussets Institute of Technology (MIT) n'est plus à démontrer [SAX, 94]. Le rôle des universités dans la réussite de certaines villes, comme Cambridge au Royaume-Uni, est largement reconnu [KIT, 04]. Si certaines universités prospèrent dans leur relation avec les acteurs d'un S.I, c'est également en raison des fonctions qu'elles assument dans leur supposé transfert de connaissances. La nature des liens avec le secteur productif, plus ou moins formelle, va déterminer le degré de transfert de connaissances, particulièrement important pour la valorisation de la recherche.

\section{Universités, Valorisation de la recherche et Système d'Innovation}

Valoriser la recherche, c'est identifier les liens et maillages créateurs de connaissances entre les laboratoires publics et les entreprises. Plus ce lien est fort, plus l'intensité systémique est marquée et permet de densifier le système d'innovation pour le rendre plus mature, plus conséquent. Tout en reconnaissant les activités de transfert comme un prolongement de sa mission fondamentale et un outil d'enrichissement de la formation des étudiants et d'appui au développement des connaissances, l'université propose à ses membres un cadre de référence pour ce type d'activité. La valorisation est ici un terme générique qui englobe toutes les pratiques qui visent à conférer aux travaux de recherche des valeurs ajoutées de nature différente. Ces pratiques de valorisation cherchent principalement à octroyer des valeurs économiques et, dans une moindre mesure, des valeurs sociales à la recherche. Mais, selon le Ministère Français de l'Enseignement (2018), l'articulation entre la sphère académique et productive n'est pas assez ancrée dans les pratiques; le manque à gagner se chiffre en centaines de milliers d'emplois, et le manque d'innovation bride le potentiel de croissance de l'économie tout entière.

Pour revenir à la genèse de ces pratiques, il est nécessaire de revenir sur la Triple Hélice des relations entre Université, Industrie et Gouvernement, qui s'est développée dans un modèle qui montre les effets des dynamiques économiques sur les transformations de leurs relations, lesquelles induisent simultanément des modifications institutionnelles à l'intérieur de chaque sphère institutionnelle [ETZ, LEY, 00]. Selon ces derniers, «des régions où il se fait moins de recherche sont maintenant bien conscientes que la science, appliquées à leurs ressources locales, constitue l'assise d'une grande partie de leur développement socio-économique dans l'avenir » (Ibid., p.147). La science émane principalement des universités mais la recherche est désormais centrée sur les problèmes définis par l'industrie où les savoirs sont également produits hors des universités. L'université devient «entrepreneuriale » et sa (troisième) mission est focalisée sur le transfert de technologique science-industrie. Cette nouvelle conceptualisation représente le fameux Mode 2 de Gibbons et al. [94] où la reconnaissance de la qualité et de l'intérêt des travaux n'est plus seulement d'ordre scientifique mais également de nature politique, sociale et commerciale. Les activités de transfert des travaux menées dans les universités vers les entreprises, au-delà de la seule communauté scientifique, ne peuvent qu'être bénéfiques à la dynamique de systèmes d'innovation. Ici, l'innovation est le résultat d'interactions fortes entre les universités, les entreprises et les administrations publiques. Pour cela, ce sont par des moyens d'échanges codifiés au système que le transfert de connaissances aura lieu. Autrement dit, le transfert est assuré par le biais d'intermédiaires et de canaux pertinents. Les intermédiaires sont des acteurs se situant au milieu 
d'autres acteurs dans des activités principales de courtage et de réseautage [AGO, YST, LEM, 13] et les canaux représentent des supports ou collaborations par lesquels ces activités peuvent être réalisées.

Faire que les entreprises entrent en relation avec les chercheurs, c'est nécessairement mettre en place une activité de coordinateur qui peut apprendre aux chercheurs à parler aux entreprises un langage qu'elles peuvent assimiler et vice versa [NAR, SIM, 06]. Cette activité de mise en réseau, est une véritable activité économique où l'incertitude est double : d'une part de la part des chercheurs en matière de financement, mais également des entreprises sur la productivité réelle de cette recherche. Comme le rappelle Barlatier et al. [16], les canaux de transmission de la recherche fondamentale vers l'industrie sont très variés : publications scientifiques, rapports, interactions formelles, conférences publiques, contrats de recherche, activités de conseil, recherche collaborative, brevets, échanges de personnels, formation d'étudiants, création de start-up académique, etc. [HOW, 06]. Parmi ces canaux, on distingue deux acteurs principaux dans une typologie proposée par Barlatier et al. (2016) qui opposent les TTOs (Technology Transfer Office) aux RTOs (Research and Technology Organizations). Le premier est relatif à une faible interaction et intervient uniquement dans l'objectif de réduire les coûts de transaction, transférer la propriété intellectuelle et sécuriser les contrats de collaboration. La seconde repose sur des interactions fortes entre la science et l'industrie, comme des partenariats de recherche, services de recherche et transfert de connaissances. Ces intermédiaires sont des acteurs importants du système d'innovation par la mise en réseau et le maillage qu'ils génèrent. Si les TTOs ne participent pas explicitement au transfert, mais agissent davantage dans la partie administrative et juridique, les RTOs, eux, s'impliquent ouvertement dans le processus de création de connaissances. Néanmoins, les RTOs et les universités ont souvent des objectifs, des axes de recherche et des programmes de recherche différents et donc les entreprises qui collaborent avec les RTOs (par rapport aux universités) ont elles-aussi des caractéristiques d'innovation différentes [GIA, BAR, PEN 19]. Mais les canaux de transfert peuvent également être informels. Ces canaux de transfert informels se réfèrent à des contacts personnels et donc à la dimension tacite du transfert de connaissances. Parmi les réseaux informels, on trouve les relations non professionnelles, les relations liées à l'enseignement et les relations professionnelles [GRO, BES, 02]. Le partage de connaissances est rarement totalement tacite ou codifié. Il se situe souvent entre les deux [NON, TAK, 95]. La codification est toutefois indispensable car la création de connaissances est un processus collectif qui suscite des mécanismes complexes de communication et de transfert. Il est alors judicieux de combiner des mécanismes de transfert formels et informels dans le lien entre les universités et les entreprises [GRI, HUS, 13].

$\mathrm{Au}$ total, les types de transfert sont plus ou moins formalisés, codifiés, marchands et institutionnalisés selon la nature de la collaboration entre les acteurs [LEV, 05]. Les plus formalisés donnent généralement lieu à un flux financier, et les plus informels sont souvent issus de relations individuelles, elles-mêmes à l'origine de contextes collectifs [GRO, BES, 02], tels que des réseaux de conférences [PER, WAL, 07]. La liste n'est pas exhaustive, mais on y trouve principalement des partenariats et services de recherche, de l'entrepreneuriat académique, du transfert de ressources humaines, de la commercialisation de propriété intellectuelle et des publications scientifiques. Néanmoins, la valorisation de la recherche en France est souvent mesurée au sens strict (part des ressources apportées aux opérateurs par les redevances sur titre de propriété intellectuelle, taux de succès des moyens accordés aux dispositifs d'incubation et de création d'entreprises), pouvant générer des tensions entre industriels et organismes publics de recherche [COR, 14].

\section{La valorisation de la recherche dans une P.M.U : le cas de I'UPJV}

Si les collaborations, canaux et acteurs sont nombreux entre la sphère académique et industrielle, c'est souvent en raison de leur représentation autour d'une université à forte notoriété ou encastrée dans un territoire dont les externalités technologiques sont importantes. A ce titre, il est aisé dans la littérature économique de retrouver des exemples de Stanford [BAR, GIA, PEN, 16] ou du MIT [SAX, 
94] pour mettre en avant les effets de synergie entre acteurs et transferts de connaissances de l'académique vers le secteur productif. A contrario, le cas des « universités en développement », telles que représentées en Amérique Latine ou en Afrique Sub-Saharienne, n'intéresse que par ses fonctions dans les activités d'enseignement, très souvent déconnectées du monde de l'entreprise [CAS, TAH, 17]. Dans le cas du système supérieur français, les Petites et Moyennes Universités ont des structures de recherche variées et une grande hétérogénéité de leur offre de formation et de valorisation.

Selon le rapport Aimé de 2016, il en existe 39 en France, marquées par des spécificités relatives à leur histoire et situation géographique, leur spécialisation, leur création et éventuellement fusion, leur activité de recherche ou leur offre de formation. La question est donc de savoir quelles sont les modalités de transfert de connaissances dans ces P.M.U, souvent fragilisées par des contraintes structurelles, matérielles, humaines, financières, plus ou moins fortes. La réponse est bien évidemment dépendante de la structure, elle-même enchâssée dans un entrelacement de compétences et de territoires, d'arrangements institutionnels et de politiques locales, régionales et nationales. Les enjeux sont nombreux dans un contexte où la dimension politique est forte et les contraintes financières réelles.

Notre méthodologie s'appuie ici sur des données secondaires issues de rapports publics ainsi que de sources internes, croisées avec une enquête qualitative réalisée début 2020 auprès des services centraux de l'université.

\subsection{Le Rapport AIME et I'UPJV}

L'UPJV apparait dans le rapport comme appartenant à l'une des 39 Petites et Moyennes Universités françaises. A sa création, en 1969, l'UPJV était l'une des rares universités à porter le nom de sa région, la Picardie. Cette mise en évidence de l'appartenance territoriale traduisait, et traduit encore, la volonté politique et universitaire d'assurer un large maillage territorial sur l'ensemble de l'ex-région Picardie et ses trois départements. La pluridisciplinarité de l'UPJV a été retenue dans cette perspective.

Si 90\% des 31000 étudiants sont inscrits sur Amiens, les 10\% restant sont répartis entre les 6 autres sites (campus) délocalisés de l'université (Cf. Figure 1). Dans ses sites, nous retrouvons des composantes à part entière, comme les IUT de l'Aisne et de l'Oise ou des antennes de composantes amiénoises comme STAPS ou l'INSPE par exemple.

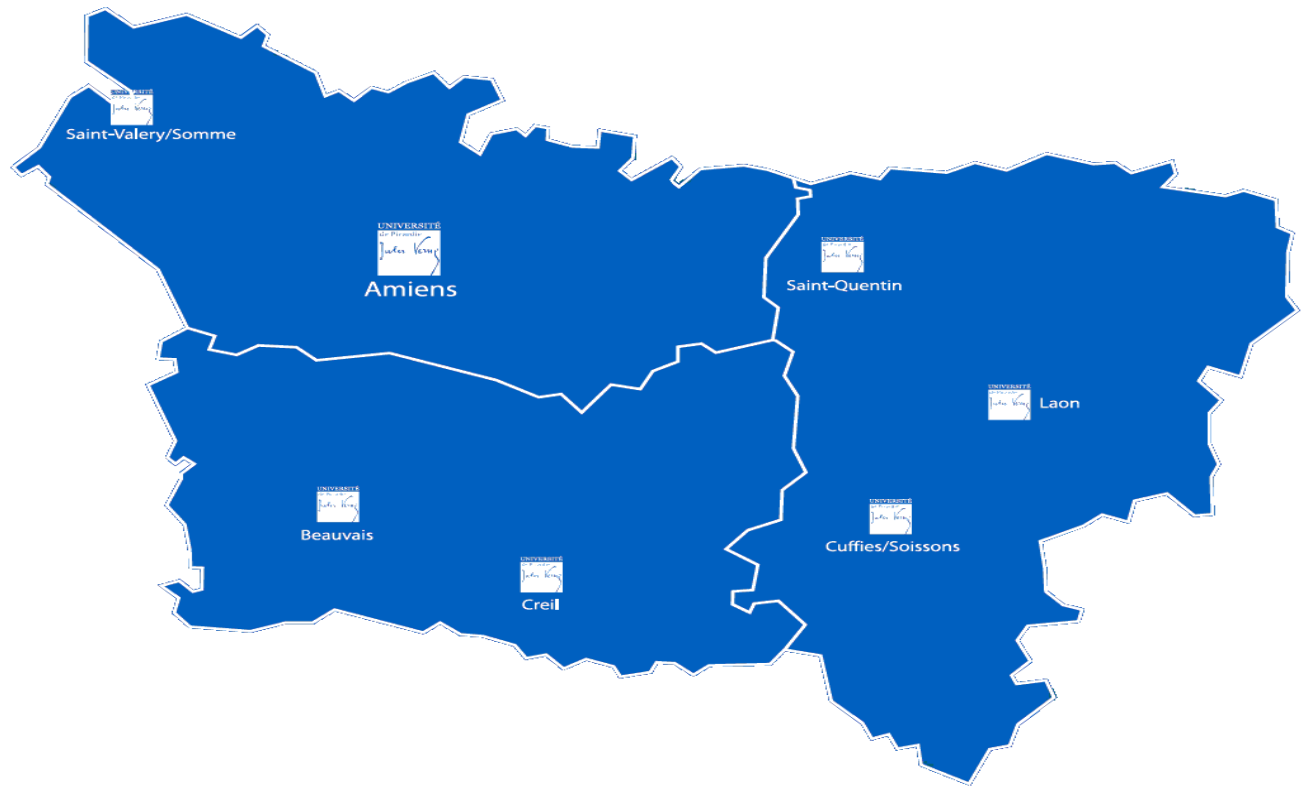

Figure 1. Les différentes implantations régionales de I'UPJV 
Le rapport Aimé de 2016 met en place une série d'indicateurs, comme l'offre de formation, la qualité de recherche ou l'ancrage territorial, et y associe une cotation (sur 5). Les indicateurs quantitatifs permettent de comparer les établissements, et d'en réaliser des typologies appropriées. Le rapport fait apparaitre un certain nombre de points positifs mais également de nombreuses contraintes liées à la formation et à la recherche. Ces contraintes peuvent être handicapantes dans l'objectif d'une importante valorisation de la recherche.

L'évolution démographique et l'ancrage territorial et social sont les meilleurs atouts de l'UPJV (à partir de critères liés à l'évolution du nombre d'étudiants, le recrutement académique, l'inscription en apprentissage ou les boursiers et bacheliers professionnels). Ce fort ancrage territorial se réalise par le biais de nombreux partenariats avec les milieux professionnels locaux, lequel explique à son tour le taux d'insertion professionnelle des diplômés de la formation, dans des emplois en adéquation avec ses objectifs. En 2020, les formations IUT de l'UPJV ont été classées 5 ème meilleures formations IUT de France. Trois critères ont été retenus pour établir ce classement : l'attractivité, le taux de réussite et la valeur ajoutée pédagogique (différence entre le taux de réussite attendu par le MESRI et celui réalisé). Sachant que ces formations IUT sont les plus délocalisées de l'université, ces résultats mettent en exergue le rôle socioéconomique de l'université dans le développement territorial régional.

La politique de partenariats est forte, que ce soit avec le CHU, les collectivités territoriales ou même les interfaces de l'environnement socio-économique. Pour ne citer que les principaux, des partenariats se sont développés avec les pôles de compétitivité I-Trans, Up-Tex et avec le pôle industrie et agro-ressources pour lequel l'UPJV est partie prenante dans les instances exécutives et décisionnelles et participe aux commissions liées à la recherche et à la formation.

Les fragilités, sont, quant à elles, assez marquantes dans ledit rapport. Les indicateurs d'efficacité de l'offre de formation montrent un certain nombre de difficultés, eu égard à d'autres établissements. Les critères associés sont inhérents aux taux de réussite, poids des Master, existence d'un IUT, secteur santé, école d'ingénieurs et ESPE ainsi que sur la part des nouveaux entrants M1, M2. L'UPJV dispose pourtant d'un secteur santé, de plusieurs IUT, d'une convention de partenariat avec l'UTC Compiègne (Université Technologique) ainsi que d'un INSPE. C'est donc dans la diversité de l'offre de formation qu'il faut entrevoir les difficultés, comme le précise le dernier rapport HCERES de l'UPJV de 2016, mettant en avant une structuration en champ de formation inaboutie et des initiatives de transformation des pratiques pédagogiques trop isolées, préfigurant un réseau à l'échelle de l'établissement.

De même, l'UPJV se situe dans un groupe d'établissements mettant en avant une bonne qualité de recherche mais une intensité relative de cette dernière. L'intensité des activités de recherche est notamment représentée par le montant des contrats de recherche, le nombre de thèses sur 3 ans, de directeurs et chargés de recherche, de publications ou de Labex et la qualité des activités de recherche est marquée, entre autres, par le montant des subventions ANR, les facteurs d'impact des publications et le nombre de thèses par enseignant-chercheur. Contrairement à l'Université Technologique de Compiègne, l'UPJV a certes un bon potentiel recherche (2 écoles doctorales, 35 unités de recherche et 6 fédérations de recherche), mais il existe un déséquilibre entre le nombre de structures regroupées en chercheurs travaillant sur une thématique commune (23) et le nombre d'unités mixtes de recherche (UMR, 10). De même, le nombre de chercheurs des organismes est aussi très faible, accompagné d'un faible nombre de doctorants (HCERES, 2016). Les financements publics de thèses se partagent entre des contrats doctoraux (État) au nombre de 15 ( 9 en STS et 6 en SHS) et 20 financements de l'ancienne région picarde (17 en STS et 3 en SHS). Enfin, la présence de sites délocalisés ne permet pas toujours la création d'une dynamique et cohérence de projets de recherche.

Au total, l'UPJV se situe dans la configuration suivante : 


\begin{tabular}{|c|c|c|c|c|}
\hline Critères & $\begin{array}{l}\text { Ancrage } \\
\text { territorial }\end{array}$ & $\begin{array}{c}\text { Efficacité de l'offre } \\
\text { de formation }\end{array}$ & $\begin{array}{l}\text { Qualité de la } \\
\text { recherche }\end{array}$ & $\begin{array}{c}\text { Intensité de la } \\
\text { recherche }\end{array}$ \\
\hline Résultats & Fort & Modérée & Disparate & Faible \\
\hline Causes & $\begin{array}{l}\text { Partenariats avec } \\
\text { les acteurs locaux } \\
\text { (interfaces de } \\
\text { l'environnement } \\
\text { socio-économique) }\end{array}$ & $\begin{array}{l}\text { Structuration des } \\
\text { champs de } \\
\text { formation inachevée } \\
\text { Grande } \\
\text { pluridisciplinarité de } \\
\text { l'offre de formation } \\
\text { Eclatement des sites } \\
\text { (sites délocalisés) }\end{array}$ & $\begin{array}{l}\text { Grande diversité } \\
\text { des équipes de } \\
\text { recherche } \\
\text { Points d'appui } \\
\text { d'excellence en } \\
\text { recherche (agro- } \\
\text { ressources, } \\
\text { mobilité et } \\
\text { énergie, santé et } \\
\text { technologie) }\end{array}$ & $\begin{array}{l}\text { Lien formation - } \\
\text { recherche peu } \\
\text { visible } \\
\text { Faible nombre de } \\
\text { chercheurs et de } \\
\text { doctorants }\end{array}$ \\
\hline
\end{tabular}

Tableau 1. Les indicateurs représentatifs des Petites et Moyennes Universités françaises Source : auteurs

\subsection{La valorisation de la recherche à I'UPJV}

La valorisation de la recherche a été mise en exergue dans un carré organique proposé par Laperche [02]. Ce carré représente quatre facteurs clés de la valorisation :

-La règlementation reposant sur les missions d'une université et la législation sur les droits de propriété intellectuelle,

-Le progrès technique basé sur l'évolution des dépenses de la R\&D,

-La stratégie université liée à la politique d'établissement, le développement d'axes stratégies et les incitations des chercheurs à la valorisation,

-Le milieu économique et esprit d'entreprise représentant l'ancrage territorial et l'effet réseau avec le milieu local.

Réglementation

- fonction publique

- mission des universités

- droits de propriété intellectuelle
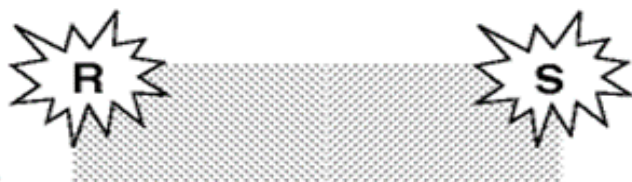

VAL ORISATION

DE LA RECHERGHE

PUBLIQUE

\section{Progrès technique}

- financement de la R-D

- avance dans les domaines

potentiellement valorisables

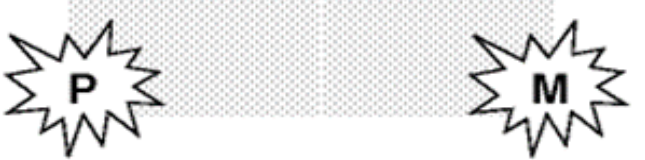

Milieu économique et esprit d'entreprise

- incitations à la création d'entreprise

- demande en matière de science

et de technologie

Figure 2. Le carré organique de la valorization

Source : LAP [02] 
Elle est représentée par l'ensemble des dispositions légales, les structures d'accompagnement et de transfert ainsi que par la direction des politiques industrielles (DPI).

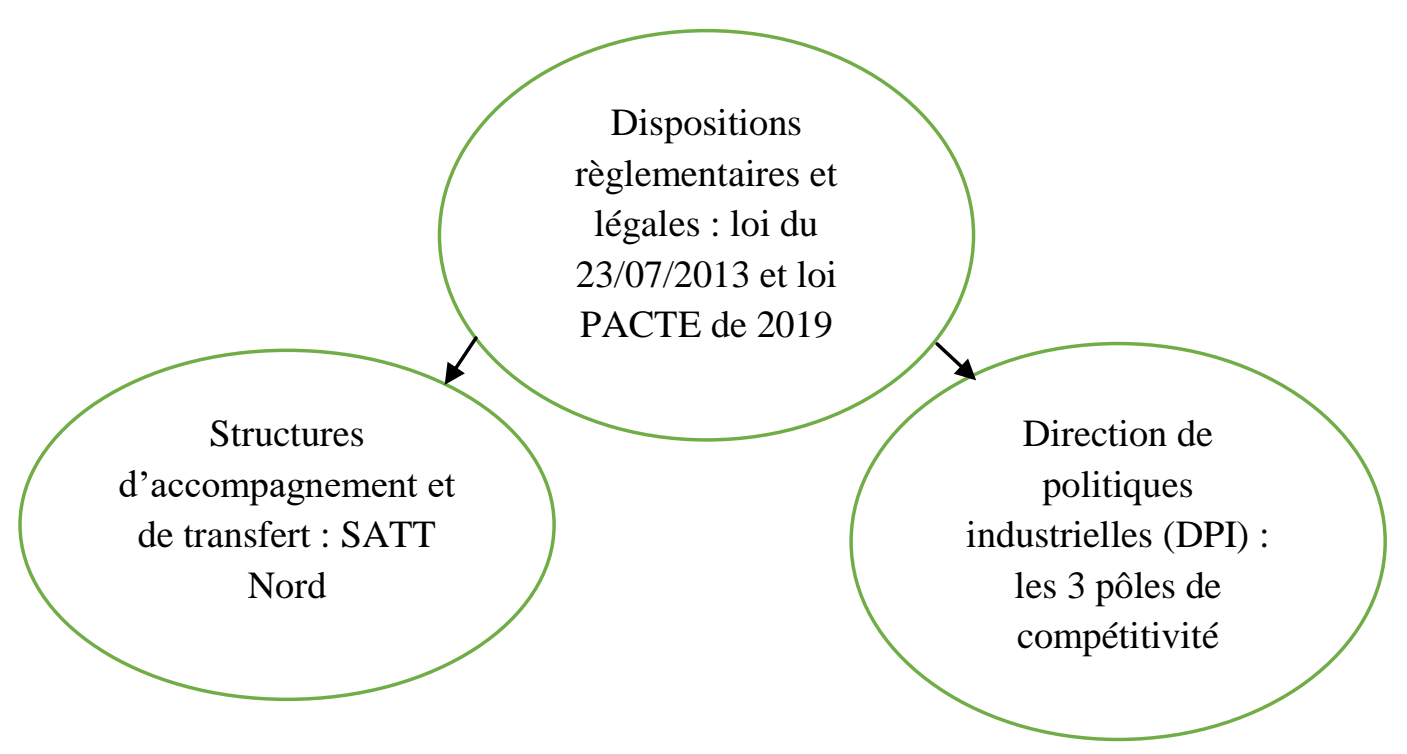

Figure 3. La règlementation de la valorisation adaptée à l'UPJV

Source : auteurs

Pour revenir sur le cadre de la règlementation de la valorisation, il faut revenir sur les travaux de Robin $[14,19]$. On citera ainsi deux principales lois :

-La loi du 22 juillet 2013 relative à l'enseignement supérieur et à la recherche,

-La loi PACTE du 22 mai 2019.

Le premier dispositif place la valorisation au service de la société. Cette loi s'attache au développement de l'innovation, du transfert de technologie lorsque celui-ci est possible, de la capacité d'expertise et d'appui aux associations et fondations, reconnues d'utilité publique, et aux politiques publiques menées pour répondre aux défis sociétaux, aux besoins sociaux, économiques et du développement durable [...] (art. 14, mod. art. L. 111-1 C.). Elle insiste sur le développement de l'innovation, dont le terme révèle la volonté du législateur et des pouvoirs publics de développer la culture de l'innovation et de l'entrepreneuriat, d'accompagner la croissance des entreprises innovantes et de mettre en place les instruments d'une politique publique de l'innovation [ROB, 14]. Quant à la loi Pacte, elle favorise la croissance par la facilitation de l'activité entrepreneuriale. Ce Plan d'Action pour la Croissance et la Transformation des Entreprises (PACTE) contient des dispositions propres au droit de la propriété intellectuelle, à la mobilité des chercheurs, agents publics, à la gestion des brevets pris en copropriété par les établissements publics, ainsi qu'à la gestion de la copropriété intellectuelle entre établissements publics [ROB, 19]. Ces deux dispositifs promeuvent et facilitent la valorisation par le biais de volets sur la protection intellectuelle protégeant ainsi les inventions, brevets et innovations. Parmi un des aspects concrets concernant le statut du chercheur, l'entreprise peut revaloriser des résultats de recherche publique que le chercheur en soit ou non l'auteur ou l'inventeur. Aussi il n'y a aucune obligation pour l'entreprise de valoriser la propriété intellectuelle issue du chercheur ou de son laboratoire d'origine : elle doit valoriser des travaux de la recherche publique sans contrainte de lien avec le chercheur ou son employeur. 
Au niveau des infrastructures, ce sont les SATT qui sont les plus représentatives au niveau des TTOs. Ces Structures d'Accompagnement du Transfert Technologique ont pour principal objectif de porter le soutien à la maturation et de favoriser le transfert vers le secteur économique des résultats issus des laboratoires publics, par le biais du dépôt de brevets puis de la concession de licences ou de la création de start-ups. Quatorze SATT ont été mises en place depuis 2012 afin de développer la valorisation de la recherche publique. Ce sont des sociétés privées (sociétés par actions simplifiées) ayant pour actionnaires, outre la Caisse des dépôts et consignations pour le compte de l'État, des établissements et des organismes de recherche. Au niveau de la SATT Nord, selon le rapport HCERES de l'UPJV de 2017, la valorisation des résultats de la recherche se traduit par une maturation de projets dynamiques : la SATT Nord a investi $20 \%$ de son enveloppe d'investissement sur des projets émanant des laboratoires de l'UPJV. Ce pourcentage est supérieur à la force de recherche des actionnaires de la SATT qui s'élève à $12 \%$ pour UPJV.

Enfin, concernant le pilotage des politiques industrielles, en 2005, le gouvernement français a lancé une «nouvelle politique industrielle » portant sur la création de pôles de compétitivité répartis sur l'ensemble du territoire national. Le gouvernement va alors financer des projets de R\&D venant des acteurs économiques privés et sélectionnés par les membres des gouvernances de ces pôles. L'UPJV est partie prenante de 3 pôles de compétitivité précités (transport, textile, bioéconomie).

\section{$\underline{\text { Le progrès technique }}$}

Le progrès technique est notamment représenté par l'évolution du financement de la R\&D. En France, si l'on constate une nette augmentation des dépenses intérieures de la R\&D depuis 1995, la part de la R\&D dans le PIB n'a pas, quant à elle, évolué. Elle tourne autour des $2 \%$, ce qui est bien loin de la Suède $(3,25 \%$ du PIB) ou de l'Allemagne (2,94\% du PIB en 2016). La moyenne européenne se situe à $2 \%$.

\begin{tabular}{|l|c|c|c|c|c|}
\hline Années & $\mathbf{1 9 9 5}$ & $\mathbf{2 0 0 0}$ & $\mathbf{2 0 0 5}$ & $\mathbf{2 0 1 0}$ & $\mathbf{2 0 1 6}$ \\
\hline Dépense intérieure de recherche et développement (DIRD) & $\mathbf{2 7 3 0 2}$ & $\mathbf{3 0 ~ 9 5 4}$ & $\mathbf{3 6 2 2 8}$ & $\mathbf{4 3 ~ 4 6 9}$ & $\mathbf{4 9 5 3 4}$ \\
\hline Exécution par les administrations & 10653 & 11605 & 13725 & 16014 & 17352 \\
\hline Exécution par les entreprises & 16649 & 19348 & 22503 & 27455 & 32181 \\
\hline Part des entreprises dans la DIRD (en \%) & 61,0 & 62,5 & 62,1 & 63,2 & 65,0 \\
\hline Part de la DIRD dans le PIB (en \%) & 2,24 & 2,09 & 2,05 & 2,18 & 2,22 \\
\hline
\end{tabular}

Tableau 2. Dépenses intérieures de R\&D en France Source : MESRI- SIES

Les activités les plus représentatives de la R\&D française sont l'industrie automobile, suivies de la construction aéronautique et spatiale ainsi que l'industrie pharmaceutique. La France se situe donc dans une haute moyenne européenne, mais ses dépenses en R\&D sont insuffisantes en ellesmêmes pour analyser la stratégie globale de la valorisation, les politiques d'établissement étant également décisives pour mettre en avant les effets de la Valorisation.

A l'UPJV, la recherche a été construite sur trois axes de spécialisation : (1) agro-ressources (bioéconomie et bio-raffinerie) ; (2) énergie et mobilité ; (3) santé et technologies. Trois axes transversaux se sont ajoutés, permettant d'associer les sciences humaines et sociales aux sciences expérimentales : (1) maîtrise des risques chroniques et accidents, et impacts environnementaux ; (2) 
développement des outils numériques et maîtrise des systèmes ; (3) promotion des SHS dans l'approche des grands défis sociétaux et innovation sociale.

\section{La stratégie universitaire}

Pour reprendre les formes de valorisation et en les associant au cas de l'UPJV, nous allons nous baser sur la présentation du financement de la recherche à l'UPJV de 2019, sur le rapport de l'HCERES de 2017, ainsi que sur une enquête qualitative réalisée auprès du VP Recherche, Directeur Général Adjoint du Pôle Recherche, 3 Directeurs de laboratoire de Recherche et une Responsable de Projets SHS. La plus grande partie des questions représente des données chiffrées et quantitatives, pour ensuite laisser place à des réponses plus ouvertes concernant les contraintes de la valorisation.

\begin{tabular}{|l|l|}
\hline \multicolumn{1}{|c|}{$\begin{array}{c}\text { Formes de collaborations entre l'UPJV et le } \\
\text { monde professionnel }\end{array}$} & \multicolumn{1}{c|}{ Données chiffrées (2019) } \\
\hline Thèses CIFRE & 13 \\
\hline Nombre de start-ups & 1 \\
\hline Nombre de projets en maturation - SATT & 1 \\
\hline Brevets & $\begin{array}{l}\text { en 2019 mais } 60 \text { brevets depuis } 2008 \text { (copropriété } \\
\text { UPJV) et } 16 \text { brevets depuis 2000 (100\% UPJV) }\end{array}$ \\
\hline Projet européen (Interreg FP7 H2020) & 9 \\
\hline $\begin{array}{l}\text { Projet local, régional ou national (ANR, Ministère, } \\
\text { Régional) }\end{array}$ & 15 \\
\hline Manifestation scientifique & 7 \\
\hline Participation à des clusters locaux & 3 \\
\hline
\end{tabular}

Tableau 3. Données chiffrées de la stratégie universitaire de valorisation de la recherche à l'UPJVr'ef Source : auteurs

Le brevet est un outil de valorisation important. Il s'agit des inventions qui sont souvent générées dans des laboratoires mixtes, par des équipes dont les membres sont indifféremment employés par l'UPJV ou d'autres organismes. Ce nombre n'a cessé de croitre depuis 20 ans, malgré sa faible représentativité au niveau national. Le nombre de thèses CIFRE est également en constante progression (8 thèses CIFRE en 2018). Quant au nombre de projets, ils sont particulièrement importants au sein d'une moyenne structure, que ce soit sous forme de projets collaboratifs ou encore de manifestations et publications scientifiques. En effet, dans le cadre du programme d'investissements d'avenir (PIA1), l'UPJV est impliquée dans plusieurs projets, notamment dans un Laboratoire d'excellence dans le domaine des sciences de la matière et de l'ingénieur (Labex STOREX), un Equipement d'excellence (Equipex FIGURES), un Institut pour la transition énergétique sur la chimie du végétal (ITE PIVERT), un Institut de recherche technologique (IRT) sur le ferroviaire (RAILENIUM), et un institut d'excellence en formation innovante (Idefi Simusanté). Le projet d'Initiatives Science - Innovation - Territoires - Économie (I-Site) « Picardie Unité : un Mouvement pour une Ambition » (PUMA) porté par l'UPJV n'a cependant pas abouti dans le cadre du PIA2. Les appels à projets (AAP) sont donc importants et montrent la volonté de 
l'UPJV de valoriser ses liens avec les partenaires extérieurs. Nous verrons par la suite que des incitations sont mises en place pour pouvoir développer les projets. Au-delà du service de veille d'AAP, on y trouve des appels internes (le S2R, soutien au rayonnement de la recherche), un projet commun avec 2 autres P.M.U (Artois et ULCO), des appels à projets régionaux (Start-AIRR, SACRE, FRAPE, EMILE), nationaux (ADEME, PRC) et internationaux (H2020, ERC, Interreg des 2 mers, Marie Curie).

Les deux acteurs de transfert sont la SATT, dont nous avons déjà donné quelques références et l'association Ombelliscience Picardie qui a comme objectif le partage des savoirs dans les domaines scientifique et technique. Son rôle est de dynamiser la coordination entre toutes les activités de ce domaine. Le dynamisme de cette structure est à souligner avec différentes initiatives visant à favoriser auprès des plus jeunes, en particulier ceux qui sont éloignés des centres de production industrielle et culturelle, une plus grande appétence pour les sciences mais également une meilleure connaissance des métiers. Depuis 2014, Ombelliscience Picardie coordonne le projet financé par le PIA « Picardie Science : l'industrie et l'innovation en partage ».

Enfin, la métropole d'Amiens a mis en place trois clusters dans le domaine de l'autonomie énergétique, le numérique, et l'e-santé, chacun impliquant des laboratoires et plates-formes de l'UPJV, et permettant de développer des liens de recherche appliquée avec les entreprises du territoire, avec une volonté de décloisonnement disciplinaire, notamment en direction des SHS.

\section{Le milieu économique et esprit d'entreprise}

Comme préalablement mentionné et en parallèle avec le rapport Aimé, l'ancrage territorial de l'UPJV est l'un de ses points forts. La politique partenariale territoriale est décliné dans un document «Projet de site : Picardie Universités » et définit trois axes stratégiques : Renforcer l'attractivité du site sur la base de projets phares, entraîner la jeunesse picarde vers la réussite et articuler les expertises académiques avec les forces du territoire. Ainsi, l'UPJV, dans sa politique partenariale de proximité, s'est affirmée, comme " établissement leader » d'un projet de site ambitieux «qui a vocation à construire une communauté fondée sur des projets et des coopérations réussis » [HCE, 16].

Sur sa politique de coopération interuniversitaire, l'UPJV entend l'élargir, en particulier mais non exclusivement, aux universités du Littoral Côte d'Opale (ULCO), d'Artois (UArtois) et de Valenciennes Hainaut-Cambrésis (UVHC), dans une logique de projets et de réseau. Il s'agit à la fois d'un élargissement géographique (au-delà du territoire picard, dans une vision régionale), d'un élargissement de contenus (recherche et formation), et d'un changement de méthode (regroupements d'universités en réseaux construits autour de projets partenariaux).

Au niveau de sa coopération avec les infrastructures publiques locales, le Conseil Régional de Picardie joue, depuis de nombreuses années, un rôle essentiel par une convention-cadre, déclinée dans des conventions annuelles et portant sur des bourses doctorales, post-doctorales, des postes de personnels techniques, des appels à projets, des prix de thèse, des financements d'équipements informatiques, des bourses de mobilité internationale, des ressources pour le Fonds de Solidarité et de Développement des Initiatives Etudiantes (FSDIE). Au cours du dernier contrat, la région a également porté le Schéma régional d'enseignement supérieur et de la recherche (Sresr), la Stratégie de spécialisation intelligente (S3) et le Contrat de Plan Régional de Développement des Formations Professionnelles (CPRDFP). Dans le cadre du Contrat de Plan État-Région (CPER) 2015-2020, la collectivité régionale s'est engagée dans la poursuite de projets immobiliers (en particulier l'opération Citadelle et le plateau technique santé) et dans l'acquisition d'équipements scientifiques et de dépenses de transfert de technologie dans les secteurs d'excellence de l'UPJV (chimie verte, stockage de l'énergie, bio-économie...). 
Enfin, sur ses relations avec le monde productif et/ou agricole local, des partenariats ont été mis en place avec des centres techniques, dans les domaines de la construction durable et les écomatériaux (CoDEM), de la chimie de la biomasse végétale (Extarsis), des polymères et matières plastiques (Critt Polymères). Un partenariat existe également avec le centre d'innovation IndustriLab qui intègre la plate-forme d'innovation Cademce. Ce partenariat permet de développer des thématiques concernant le transport ferroviaire. Par ailleurs, l'UPJV est membre fondateur de l'Institut pour la transition énergétique "Picardie innovations végétales : enseignements et recherches technologiques » (ITE - PIVERT), lauréat dans le cadre du PIA1. PIVERT est associé au programme de recherche GENESYS qui vise à déterminer les bases de la raffinerie oléagineuse du futur et qui est actuellement dirigé par une enseignante-chercheuse de l'UPJV. L'UPJV est également membre fondateur de la SAS IMPROVE, plate-forme dédiée à la valorisation des protéines végétales, et hébergée dans l'établissement.

L'ensemble de ces partenariats témoigne de l'intégration de l'UPJV dans son territoire et de ses liens avec ses interfaces socio-économiques, en particulier autour des thématiques d'excellence que sont la bio-économie, le transport et l'énergie.

\begin{tabular}{|c|c|c|c|}
\hline $\begin{array}{c}\text { Types de } \\
\text { collaborations }\end{array}$ & $\begin{array}{c}\text { Coopérations inter- } \\
\text { universitaires }\end{array}$ & $\begin{array}{c}\text { Collaborations avec } \\
\text { des infrastructures } \\
\text { publiques }\end{array}$ & $\begin{array}{c}\text { Collaborations avec } \\
\text { des partenaires de } \\
\text { l'environnement socio- } \\
\text { économique }\end{array}$ \\
\hline Supports & $\begin{array}{c}\text { Projets / recherche et } \\
\text { formation }\end{array}$ & $\begin{array}{c}\text { Bourses, AAP, prix de } \\
\text { thèse, équipements } \\
\text { scientifiques, } \\
\text { informatiques }\end{array}$ & $\begin{array}{c}\text { Partenariats formels } \\
\text { avec le secteur } \\
\text { industriel ou agricole }\end{array}$ \\
\hline
\end{tabular}

Tableau 4. Les supports de l'ancrage territorial de I'UPJV

Source : auteurs

\section{Les contraintes de la valorisation}

A la lecture des différents rapports et eu égard à nos divers entretiens, nous pouvons constater trois principales contraintes à la valorisation de l'UPJV :

-Le sentiment d'isolement et la difficulté des équipes de recherche à monter différents projets (par absence de temps ou de compétences adaptées). C'est en cela que l'UPJV a répondu à cette problématique en modifiant le REH (Référentiel Equivalent Horaire) des enseignants chercheurs pour le dépôt de projet, le portage de projet, mais également par la contribution au sein du Réseau VP Recherche pour réfléchir à l'incitation à l'entrepreneuriat, ou le développement d'une politique conjointe formation -recherche pour la mise en place de pôles à l'étranger. Une restructuration entière de la Direction de la valorisation de la recherche a été également réalisée, cette dernière s'engageant à aider plus précisément les porteurs de projets.

-Le manque de connexions entre les différents établissements de l'Association Picardie Universités (APU) pour répondre à divers AAP. Le Rapport Aimé avait pointé l'incohérence de la politique de site, sans projet et avec des financements précaires. L'élargissement de la politique de site sonnait comme une évidence à instaurer rapidement. L'UPJV a ainsi bien réagi par ses propositions de coopérations interuniversitaires avec l'ULCO et l'Artois (telles que décrites dans la partie ancrage territorial). Pour mieux comprendre l'APU et le graphique 
ci-dessous, dès la loi de $2013^{1}$, l'Université de Reims, avec laquelle l'UPJV avait instauré un rapprochement durable, avait choisi de créer une COMUE avec l'Université de Technologie de Troyes (UTT), tandis que l'UTC de Compiègne choisissait de participer à la COMUE Sorbonne Universités, excluant par là toute possibilité d'envisager une COMUE avec l'UPJV. Ainsi, fortement contrainte dans ses choix, l'UPJV s'est trouvée naturellement conduite vers la création d'une Association Picardie Universités (APU). Cette association est un regroupement souple favorisant la logique de projets et laissant à chaque établissement sa complète autonomie, juridique et financière. Ses membres sont : l'UTC, l'Esiee, l'Esad, l'Escom, UniLaSalle de Beauvais.

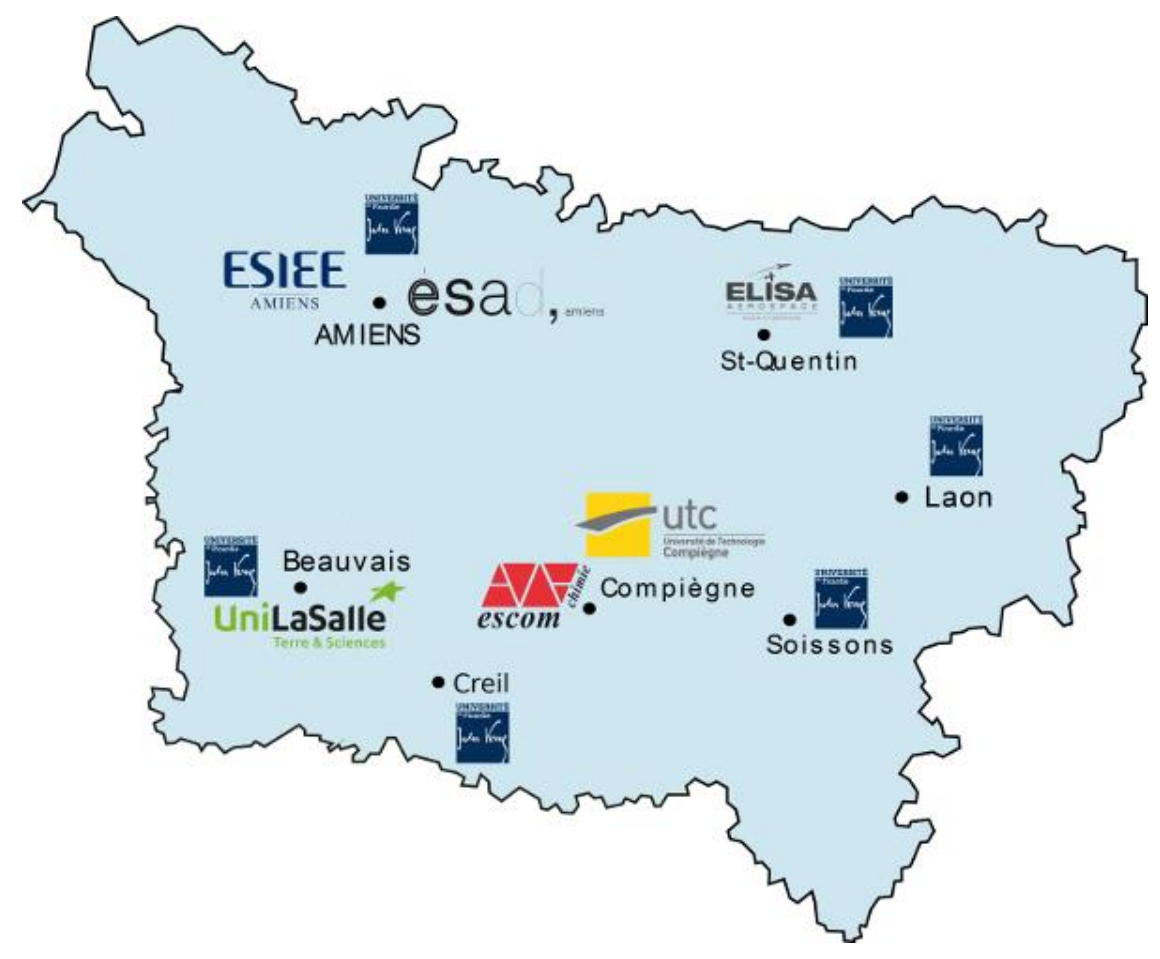

Figure 4. L'APU Picardie

Source : www.u-picardie.fr/apiu

-Le rôle parfois déconnecté du système académique des interfaces de transfert. Le rôle peu dynamique de l'incubateur régional est un frein au dynamisme du transfert même si la SATT Nord joue un rôle important dans la maturation des projets. Les domaines de la santé et du stockage électrochimique de l'énergie sont extrêmement porteurs et représentent les liens les plus forts avec les organismes de recherche nationaux (Inserm, Ineris et CNRS) qui ont contribué à l'obtention de projets phares au cours du PIA de l'UPJV (Labex STOREX, Equipex FIGURES) mais leur mise en œuvre est parfois contrastée en raison de l'absence de liens concrets avec les interfaces du monde socio-économique. Là encore, l'UPJV a instauré un nouveau pôle « Direction des Partenariats et des Projets Stratégiques » (D2PS) afin de favoriser la mise en synergie des forces du territoire et amplifier leur lisibilité/visibilité.

\footnotetext{
${ }^{1}$ La loi n²013-660 du 22 juillet 2013 relative à l'enseignement supérieur et à la recherche prévoit trois modes de regroupement des établissements d'enseignement supérieur sur un site : la création d'un nouveau EPSCP par fusion, la participation à une communauté d'universités et établissements (COMUE), et l'association d'établissements et organismes publics et privés à un EPSCP. L'UPJV a choisi la dernière proposition.
} 


\begin{tabular}{|l|l|}
\hline \multicolumn{1}{|c|}{ Contraintes } & \multicolumn{1}{|c|}{ Solutions - Incitations } \\
\hline Isolement/Difficultés des & Incitations valorisées dans le \\
équipes de recherche à monter & R.E.H (montage et porteur des \\
des AAP & $\begin{array}{l}\text { dossiers) } \\
\text { Accompagnement plus précis } \\
\text { dans le montage des AAP }\end{array}$ \\
\hline $\begin{array}{l}\text { Manque de connexions avec les } \\
\text { autres établissements publics } \\
\text { de Picardie }\end{array}$ & $\begin{array}{l}\text { Proposition de collaborations } \\
\text { interuniversitaires } \\
\text { Elargissement de la politique } \\
\text { de site }\end{array}$ \\
\hline $\begin{array}{l}\text { Manque de dynamisme des } \\
\text { interfaces de transport }\end{array}$ & Nouveau pôle D2PS \\
\hline
\end{tabular}

Tableau 5. Contraintes et Solutions de la valorisation à l'UPJV Source : auteurs

\section{Les spécificités de la valorisation de la recherche dans une P.M.U et son impact sur son Système d'Innovation}

\subsection{Les spécificités de la Valorisation dans une P.M.U}

Dans le cas de l'UPJV, et d'une P.M.U en général, un certain nombre de spécificités sont à préciser sur la valorisation. Ces spécificités sont liées aux contraintes financières, matérielles et humaines de toute petite et moyenne structure. Au niveau des contraintes humaines, le rapport UPJV de 2016 faisait état d'un taux d'encadrement pédagogique inférieur de 4 points à la moyenne nationale, avec ses 1261 enseignants titulaires et une évolution démographique très forte (23000 étudiants en 2010 et plus de 31000 en 2019). Dès 2016, la Cour des comptes a d'ailleurs alerté sur la situation financière de quinze des soixante-dix universités françaises. L'UPJV y est inscrite comme telle. Elle est sous-dotée en personnel : elle a, à titre d'exemple, 50\% de dotation en moins (en postes) par rapport à une université française de taille comparable. Dans un contexte de hausse massive des effectifs et sans dotation supplémentaire de l'Etat pour y faire face, il faut composer avec ses propres moyens. Dès lors, à moyens constants, si certains types de collaborations se sont trouvées trop onéreuses (licences/brevets), c'est dans la forte contribution à appel à projets ainsi que dans le renforcement des relations de proximité qu'il a fallu trouver un avantage compétitif.

Un cercle vertueux de la valorisation peut en ce sens être dessiné : les AAP et projets renforcent l'ancrage territorial (notamment avec les AAP internes ou régionaux), mettant en exergue la bonne qualité de recherche de l'établissement; ces trois qualités (AAP dynamiques/Ancrage territorial et qualité de recherche) ayant été précédemment relevées. Néanmoins, les projets n'ont de sens qu'avec la personnalité des universitaires s'ils sont les initiateurs des projets [BOL, SCH, CAD, 13]. La prochaine étape est de profiter de l'évolution démographique de l'UPJV pour augmenter le nombre de doctorants (notamment dans les domaines d'excellence précités) afin de palier (en partie) le problème de l'intensité de la recherche, elle-même dépendante des contraintes budgétaires de l'UPJV. 
AAP

recherché

Personnalité

des universitaires

spécialisation
Contrats/projets de recherche :

Ancrage territorial renforcé
Augmentation de la

qualité de la recherche

Effet de spécialisation

sur des secteurs de référence

Figure 5. Cercle vertueux de la valorisation à l'UPJV

Source : auteurs

\subsection{Le carré organique d'une P.M.U dans le S.R.I où le besoin d'interprétation du transfert science-industrie au sens large}

En matière de relations entre établissements d'enseignement et de recherche et industrie, qu'il s'agisse de chercheurs ou de pouvoirs publics, l'attention se polarise très souvent sur le transfert de technologies, soit via des licences, soit via la création d'entreprises [COR, 14]. Les efforts des pouvoirs publics pour faire évoluer le système d'innovation français se sont donc surtout concentrés sur le transfert technologique au sens strict. Les causes sont diverses: disponibilité des données [PER, WAL, 07], vision simpliste de l'innovation reposant sur l'invention [COR, 14] mais aussi focalisation des études au sein des grandes universités [LAP, FOU, 17]. Or, dans un contexte de petite et moyenne structure, les types de collaborations peuvent être variés à partir du moment où elles créent un effet de levier en terme d'effets de proximité ou d'ancrage territorial. Genet [07] montre l'exemple d'une prestation basique de services, qui a engendré la création d'un réseau d'acteurs, la signature d'un contrat de recherche et le lancement d'un programme de R\&D. Nous avons préalablement listé les différents supports de cet ancrage à l'UPJV. Non seulement la formation des étudiants est importante par les capacités d'absorption des entreprises, mais à partir du moment où de nouvelles incitations internes, de nouvelles directions de recherche, de nouvelles veilles informationnelles ou collaborations industrielles se développent, avec l'aide d'interfaces que sont la SATT, les incubateurs ou les structures associatives, le transfert de connaissances au sens large prend du sens, qu'il soit codifié ou pas, dans un contexte de forte autonomie des universités et de ressources restreintes. Par ailleurs, si certaines universités ont pu directement externaliser leur service de valorisation (Cas de CAPACITES SAS Nantes, [BOL, SCH, CAD, 13], ou même encore VALUTEC SA pour l'Université Polytechnique Hauts de France) et ont obtenu de meilleurs résultats sur les effets (quantitatifs) de leur valorisation, d'autres n'ont pas eu la possibilité de le faire, sans pour autant préjuger des effets sur l'emploi local et le développement économique. Preuve en est avec certaines activités sous-estimées voire ignorées mais tout autant pertinentes [GRI, KEN, SIE, WRI, 11]. C'est le cas de l'UPJV avec la dynamique des projets et contrats de recherche. Les résultats en termes d'ancrage étant conséquents, la valorisation de la recherche a eu ses effets, grâce au milieu économique, à la dynamique des collaborations, partenariats et contrats de recherche. Ces contrats apportent des sources de financement, permettent la création de réseaux scientifiques et techniques et le renforcement de la notoriété du laboratoire [LAP, 02].

Représenté à travers le S.R.I, le carré organique inclut les modes de transfert (stricts ou larges), mais se définit surtout à travers un contexte sociétal diversifié [BRU, GOR, AGR,11] notamment à travers le rôle des politiques de formation, de concurrence et de S\&T, des dispositifs institutionnels ou des infrastructures de communication. 


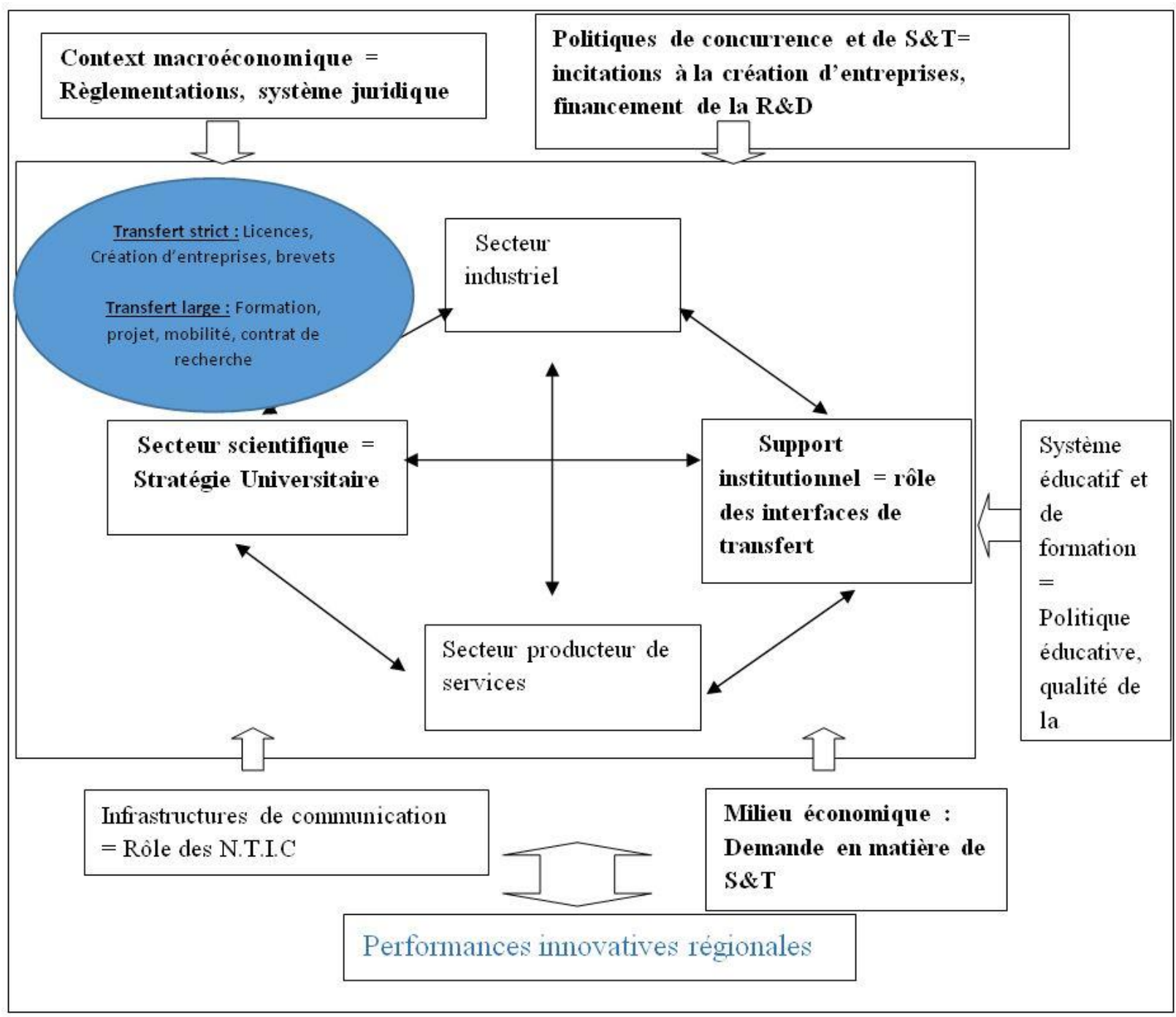

Figure 6. L'insertion du carré organique de la valorisation dans le S.R.I

Source : Auteurs

\section{Conclusion}

La valorisation n'est pas un luxe pour l'UPJV et il semble indéniable que l'activité de valorisation est un atout pour la stratégie d'une petite et moyenne Université. A notre égard, cette activité est un avantage pour deux raisons principales :

-Sur un plan microéconomique et dans un contexte actuel difficile en matière de financement des universités, un rapprochement avec l'industrie lui permet d'accroître ses capacités financières. Par exemple, à travers les thèses CIFRE, non seulement les doctorants servent de médiateurs entre les entreprises et les universités [LEV, 05] mais ils stimulent les connaissances entre le privé et le public tout en profitant des installations à moindre coût. Cette motivation engendre une autre motivation liée à l'insertion professionnelle des étudiants et aux offres d'emploi que le milieu industriel (ou agricole) peut proposer. Ces offres enrichissent le milieu économique et renforcent les effets de proximité.

-A une échelle macroéconomique, une autre motivation renvoie à la conception de l'université et son rôle dans le S.R.I. La volonté des universités est désormais tournée vers leur rôle dans la société. En ce sens, par une collaboration avec les milieux industriels, les chercheurs universitaires contribuent à enrichir le S.R.I. On retrouve alors la «troisième mission » de 
l'université qu'est le partage de connaissances et la contribution au développement global [YUS, NAB, 07]. Rôle d'autant plus important dans les P.M.U qui doivent sans cesse renouveler et modifier leurs formes de collaboration pour créer une identité et transformer leurs capacités en réelles compétences sur leur territoire. Réciproquement, si les Universités doivent s'ouvrir sur la société, le contexte macro et méso économique est également essentiel par les effets sur les incitations, la demande, les nouvelles technologies ou le financement de la $R \& D$, etc.

Au sein de l'UPJV, comme énoncé, la coopération scientifique est largement plus importante que l'exploitation des résultats de la recherche par le dépôt de brevets, la mobilité des chercheurs ou encore la création d'entreprises. Bien que le dépôt de brevets, à large échelle, reste encourageant et loin d'être négligeable depuis une vingtaine d'années, la création d'entreprises par le biais des startups peine à s'imposer. On l'a compris, le transfert « strict » n'est pas la source première de diffusion de connaissances dans une P.M.U. Il existe une variété étendue d'activités de valorisation des plus entrepreneuriales, aux plus académiques [PHI, DOO, ORE, LUP, 11]. A l'UPJV, ce n'est pourtant pas faute d'avoir initié des dispositifs, tels que Pepite, pour permettre la diffusion de la culture entrepreneuriale auprès des jeunes dans l'enseignement supérieur. Si le rapport 2017 HCERES de l'UPJV décrit l'adoption d'une politique volontariste via l'entrepreneuriat en exposant l'augmentation du nombre d'étudiants entrepreneurs (10 en 2015, 61 en 2017), cette politique a toutefois des difficultés à trouver une réelle «articulation entre ses projets spécifiques et le dispositif Pepite » [HCE, 17].

Il n'en reste pas moins à mettre en exergue deux évidences pour l'UPJV. concernant la valorisation de la recherche, au-delà et au gré des contraintes évidentes qu'il n'est plus utile de rappeler :

-La quantité de ses projets, contrats et collaborations de recherche en rapport avec ses domaines d'excellence et son milieu économique, demeure sa force principale ; Un cercle vertueux tel que précédemment mentionné est révélateur de ses pratiques actuelles ;

-Ses effets sur le S.RI par le renforcement des liens qu'elle génère au niveau de la qualité de la recherche ou les collaborations qu'elle multiplie à cet effet par le biais des pôles de compétitivité ou clusters locaux. La SATT Nord, Ombelliscience, sont des acteurs principaux pour promouvoir, coordonner et sécuriser les contrats établis à cet effet. Mais plus largement, c'est aussi le rôle des marchés du travail, de la demande et les politiques de concurrence et de S\&T qui jouent un rôle sur les pratiques de valorisation locales. Si l'université est un acteur indispensable dans le S.R.I, le système d'innovation l'est autant sur elle.

\section{Annexe 1}

\section{Réalisation de l'enquête quantitative et qualitative sur la valorisation de la Recherche à I'UPJV}

\section{I/ Eléments quantitatifs liés au contexte de valorisation}

-Nombre de doctorants, équipes de recherche, personnels de recherche

-Subventions

-Gouvernance

II/ Valorisation stricte du transfert de connaissances 
-Brevets

-Licences

-Start-ups

-Rapport avec la SATT (maturation du projet)

\section{III/ Valorisation large du transfert de connaissances}

-Nombre de contrats de recherche

-Identification des partenaires de recherche

-Sources (locales, régionales, nationales, internationales)

-Evolution des contrats sur 2 ans

-Prestation de conseil

-Thèses CIFRE

-Manifestations scientifiques (et publications)

-Clusters locaux

-Mobilité de personnels

-Rattachement à des pôles de compétitivité

\section{$\underline{\text { IV/ Contraintes liées à la valorisation (questions ouvertes) }}$}

-Financières

-Matérielles

-Techniques

-Culturelles

-Autres

\section{Bibliographie}

[AGO, YST, LEM, 13] AGOGUE M., YSTROM A., LE MASSON P., "Rethinking the Role of Intermediaries as an Architect of Collective Exploration and Creation of Knowledge in Open Innovation", International Journal of Innovation Management, 17(2), 1-24, 2013.

[BAR, GIA, PEN, 16] BARLATIER J.P, GIANNOPOULOU E., PENIN J., «Les intermédiaires de l'innovation ouverte entre gestion de l'information et gestion des connaissances : le cas de la valorisation de la recherche publique », Innovations, vol. 49, no. 1, 55-77, 2016.

[BOL, SCH, CAD, 13] BOLDRINI J.C, SCHIEB-BIENFAIT N., CADIOU J.C, «Vers de nouvelles voies de rapprochement entre recherche universitaire et PME. Etude de cas à partir d'un dispositif organisationnel innovant". XXII Conférence internationale de management stratégique AIMS. 26p., 2013.

[BRU, GOR, AGR, 11] BRUNDENIUS C., GORANSSON B., AGREN J., "The Role of Academic Institutions in the National System of Innovation and the Debate in Sweden", GORANSSON B., BRUNDENIUS C. (editors). Universities in Transition. The Changing Role and Challenges for Academic Institutions. Springer. 307-328, 2011. 
[CAR, CAM, 17] CARAYANNIS E., CAMPBELL D., « Les systèmes d'innovation de la quadruple et de la quintuple hélice », Innovations, vol. 54, no. 3, 173-195, 2017.

[CAS, TAH, 17] CASADELLA V., TAHI S., «Capacités et politiques d'innovation dans les pays moins avancés : enseignements tirés du cas du Sénégal », Innovations, vol. 53, no. 2, 13-39, 2017.

\section{CASADELLA,}

[CAS, MAT, LAS, 14] CASSIOLATO J.E, MATOS M.P, LASTRES H.M., "Innovation systems and development", CURRIE-ALDER B., KANBUR R., MALONE D.M, International development: ideas, experience and prospects, Oxford University Press, 2014.

[CAS, HAL, 94] CASTELLS M., HALL P., "Technopoles of the World: the making of the twenty first century industrial complexes", Routledge, 275p., 1994.

[COR, 14] CORBEL P., "Le rôle de la recherche et de la formation dans le SNI français », BOUTILLIER S., FOREST J., GALLAUD D., LAPERCHE B., TEMRI L., Principes d'économie de l'innovation, P.I.E. Peter Lang, 403-414, 2014.

[COO, 92] COOKE P.. "Regional innovation systems: Competitive regulation in the new Europe", Geoforum, 23, 365-382, 1992.

[COO, URA, UTX, 01] COOKE P., URANGA M.G, UTXEBARRIA G.."Regional Innovation Systems: institutionnal and organisational dimensions", Research Policy, 26 : 475-491, 2001

[DOR, BIT, 05] DOLOREUX D., BITARD P., «Les systèmes régionaux d'innovation : discussion critique », Géographie, économie, société, vol. 7, no. 1, 21-36, 2005.

[DJE, GAL, 18] DJELLAL F., GALLOUJ F., "Les réseaux d'innovation public-privé dans les services (RIPPS) : une nouvelle expression des réseaux d'innovation dans une économie des services et du développement durable ", Revue d'économie industrielle, vol. 161, no. 1, 67-118. , 2018

[LEY, ETZ, 00] LEYDESDORFF L., ETZKOWITZ H., "Le « Mode 2 » et la globalisation des systèmes d'innovation « nationaux » : le modèle à Triple hélice des relations entre université, industrie et gouvernement ». Sociologie et sociétés, 32 (1), 135-156. https://doi.org/10.7202/001434ar, 2000.

[GAL, NAD, 16] GALLIANO D., NADEL S., "Les processus sectoriels de l'innovation environnementale : les spécificités des firmes agroalimentaires françaises », Économie rurale, vol. 356, no. 6, 47-67., 2016.

[GIA, BAR, PEN, 19] GIANNOPOULOU E., BARLATIER J.P., PENIN J., "Same but different ? Research and technology organizations, universities and the innovation activities of firms", Research Policy, 48, 2019, 223-233, 2019.

[GIB, LIM, NOW, 94] GIBBONS M., LIMOGES C., NOWOTNY H.., The New Production of Knowledge, The dynamics of science and research in contemporaries' societies, Sage, London, 1994.

[GRIM, KEN, SIE, WRI, 11] GRIMALDI R., KENNEY M., SIEGEL D.S, WRIGHT M., "30 years after Bayh-Dole : Reassessing academic entrepreneurship", Research Policy, 40 : 8, 1045-1057, 2011.

[GRI, HUS, 13] GRIMPE C., HUSSINGER K., "Formal and Informal Knowledge and Technology Transfer from Academia to Industry: Complementarity Effects and Innovation Performance", Industry and Innovation, vol. 20, issue $8,683-700,2013$

[GRO, BES, 02] GROSSETTI M., BES M., «Proximité spatiale et relations science-industrie: savoirs tacites ou encastrement (Polanyi ou Polanyi) », Revue d'Economie Regionale et Urbaine, 5, 777-787, 2002.

[HOW, 06] HOWELLS J., "Intermediation and the role of intermediation in innovation", Research policy, 35, p. 715728, 2006.

[KIT, 04] KITAGAWA F., "Les universités et l'innovation dans l'économie du savoir : l'expérience des régions anglaises ». Politiques et gestion de l'enseignement supérieur, vol.16,3, 61-88, 2004.

[LAP, 02] LAPERCHE B., Le carré organique de la valorisation de la recherche. Le cas d'une jeune université dans un contexte de crise, Politiques et Gestion de l'enseignement supérieur, 2002/3, n¹4, 171-198, 2002.

[LAP, FOU, 17] LAPERCHE B., FOURMENTIN S., Enquête sur la valorisation de La recherche 2017 - Bilan ULCO, ULCO, 2017.

[LEV, 05] LEVY R., «Les doctorants CIFRE: médiateurs entre laboratoires de recherche universitaires et entreprises », Revue d'Economie Industrielle, Vol. 111, 3eme trimestre 2005, 79-95, 2005. 
[LUN, CHR, 99] LUNDVALL B.A, CHRISTENSEN J.L, "Extending and deepening the analysis of innovation systems- with empirical illustrations from the DISCO project", DRUID Working Paper N 99-12. Aalborg University. Danemark, 1999.

[LUN, JOH, AND, DAL, 02] LUNDVALL B.A, JOHNSON B., ANDERSEN E.S, DALUM B., "National systems of production, innovation and competence building", Research Policy, Vol.31, Issue 2, February 2002, 213-231, 2002.

[MOW, NEL, SAM, 01] MOWERY D.C, NELSON R., SAMPAT B.N., "The Growth of Patenting and Licensing by U.S. Universities: an Assessment of the Effects of the Bayh-Dole Act of 1980", Research Policy, 2001, 30, 99-119, 2001.

[NAY, SIM, 06] NAYARADOU M., SIMART V., 2006, «La collaboration université/entreprise : le cas du management de la recherche aux États-Unis », Vie \& sciences de l'entreprise, vol. 170 - 171, no. 1, 153-174, 2006.

[NIO, 02] NIOSI J., 2002, National Systems of Innovation are "x-efficient"- Why some are slow learners, Research Policy, 31 (2), 214-302, 2002.

[NON, TAK, 95] NONAKA I., TAKEUCHI H., The knowledge-creating company. How Japanese companies create the dynamics of innovation. Oxford university press, New York, Oxford, 1995.

[PAT, PAV, 94] PATEL P., PAVITT K., "National Innovation systems: why they are important, and how they might be measured and compared", Economics of innovation and new technology, 3, 77-95, 1994.

[PER, WAL, 07] PERKMANN M., WALSH K., "University-industry relationships and open innovation: toward a research agenda”, International Journal of Management Review, 9(4), 259-280, 2007

[PHI, DOO, ORE, LUP, 11] PHILPOTT K., DOOLEY L., O REILLY C., LUPTON G., "The entrepreneurial university : Examining the underlying academic tensions", Technovation, $31:$ 4, 161-170, 2011

[HCE,17] RAPPORT HCERES, Université Picardie Jules Verne, Disponible sur https://www.hceres.fr/sites/default/files/media/publications/depot-evaluations/C2018-EV-0801344B-DEEETAB180015373-021394-RD.pdf, 2017.

[RAP, 17] RAPPORT GUILLAUME, Rapport sur la valorisation de la recherche, 250p, 2007

[ROB, 14] ROBIN A., Valorisation de la recherche publique, innovation, propriété intellectuelle, Cahiers Droit, Sciences \& Technologies, URL : http://journals.openedition.org/cdst/348, 2014

[ROB, 19] ROBIN A., 2019, Valorisation de la recherche scientifique, propriété intellectuelle, innovation », Cahiers Droit, Sciences \& Technologies, 9, 2019, 147-157, 2019

[SAX, 94] SAXENIAN A., Regional Advantage: Culture and Competition in Silicon Valley and Route 128. Cambridge, MA: Harvard University Press, 1994.

[SCH, 19] SCHAEFFER V. "L'université entrepreneuriale : éléments historiques et débats », Marché et organisations, vol. 34, no. 1, 87-108, 2019

[SUI, VIC, 15] SUIRE R., VICENTE J., 2015, «Récents enseignements de la théorie des réseaux en faveur de la politique et du management des clusters », Revue d'économie industrielle, vol. 152, no. 4, 91-119.,2015.

[YUS, NAB, 07] YUSUF S., NABESHIMA K., How universities promote economic growth, The World Bank, 286p., 2007 